\title{
FASTEN: EU-Brazil cooperation in loT for manufacturing. The Embraer use
}

\author{
Ricardo Reis ${ }^{1 *}$, Flávio Diniz ${ }^{2}$, Luciana Mizioka ${ }^{2}$, Rosana Yamasaki ${ }^{2}$, Gléverson Lemos ${ }^{2}$, \\ Marta Quintiães ${ }^{1}$, Ruben Menezes ${ }^{1}$, Narciso Caldas ${ }^{3}$, Roberto Vita ${ }^{3}$, Ralph Schultz ${ }^{4}$, Rafael \\ Arrais $^{3}$ and Ariane Pereira ${ }^{5}$ \\ ${ }^{1}$ Embraer Engineering and Technology Center, Embraer Portugal SA, PIAE Lote A1, Herdade do \\ Pinheiro e Casa Branca, 7005-797 Évora, Portugal \\ ${ }^{2}$ Embraer SA, Avenida Brigadeiro Faria Lima, 2170, CEP 12227-901, São José dos Campos, Brasil \\ ${ }^{3}$ INES-TEC, R. Dr. Roberto Frias, 4200-465 Porto, Portugal \\ ${ }^{4}$ PACE Aerospace Engineering and Information Technology GmbH - a TXT company, Am Bahnhof \\ Westend 13, 14059 Berlin, Germany \\ ${ }^{5}$ INESC P\&D Brasil Rua José Caballero, 15, Bairro Gonzaga, 11055-300 Santos, SP, Brasil
}

\begin{abstract}
FASTEN is an H2020 project under a bilateral call UE-Brazil. Embraer is a global aerospace company, with manufacturing and assembly lines in Europe, Brazil and USA. FASTEN aims to advance IoT and IoT enabled applications to support Industry 4.0 concepts, namely in the area of automation and additive manufacturing. The project results will be demonstrated through two pilots: one in Brazil, lead by a ThyssenKrupp use case, and the other in Europe, at Embraer facilities in Portugal. The project results for the Embraer use case will be presented, with emphasis on bilateral collaboration gains provided by exploiting common frameworks for development and open architecture, and future opportunities for exploitation discussed.
\end{abstract}

\section{Motivation and FASTEN objectives}

Adopted as part of strategic initiative by German government in 2011, the 'Industrie 4.0' [1] has become a global innovation paradigm for the manufacturing industry worldwide, overcoming the increasingly competitive markets, by emerging information technologies application, defined as enablers, such as Industrial Internet of Things (IIoT), big data, cloud computing and artificial intelligence. Based on a production oriented to Cyber-Physical Systems (CPS) concept, enablers allow in achieving vertical integration of production facilities, warehousing and logistics systems [2].

As a leading aircraft Original Equipment Manufacturer (OEM), Embraer is characterized by design and manufacture of highly complex and customizable products. As such, Industry 4.0 is a key concept to maintain its leading position. Embraer global footprint encourages the adoption of global, systemic, digital integration initiatives. In Embraer environment, a complex web of machines, corporate and specific systems exist and interact, with different levels of integration. Adopting open-architectures and middleware, able to connect this diverse array of entities, allows the mitigation of vendor lock and leverages common efforts being brought by the aerospace and other industries as a 
whole. In that regard, the ability to develop these capabilities across Europe and Brazil within Horizon 2020 is a highly prized feature, as it accelerates the potential adoption of technologies across the company and creates a RD web spanning both geographical areas.

FASTEN is a H2020 under a bilateral call with Brazil that aims to realize, through two industrial lead pilots, to realize some of the high level concepts within industry 4.0, namely regarding IIoT, automation (Embraer) and 3D manufacturing (ThyssenKrupp). These pilots will test the developed solutions and technologies in an representative operational context, accelerating their understanding, and contribute to the adoption of common standards and architectures. This paper is a follow up of previous [4], presenting high level intermediate results of the FASTEN project related to Embraer Use case and mainly an addressing the exploitation of the experience between Europe and Brazil.

\subsection{Embraer Industry 4.0 strategy}

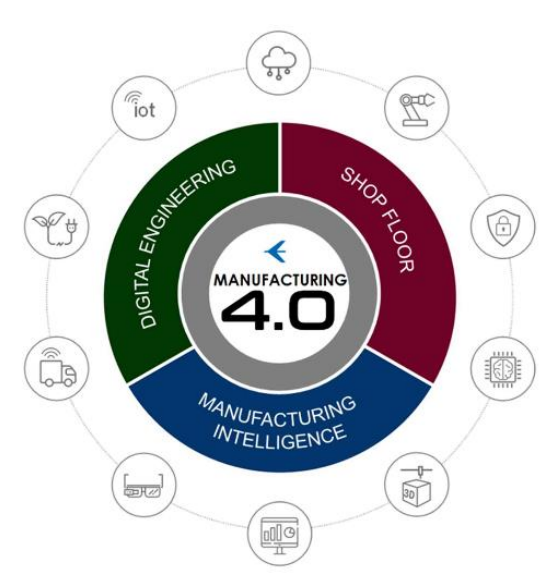

Fig.1 Embraer Industry 4.0 strategic vision
For many years, Embraer has been developing technologies to increase its productivity and flexibility, based on a centralized strategy aiming to increase its competitiveness. For instance, Embraer has invested on manufacturing processes automation, implementing around a hundred of drilling, riveting and painting robots in the assembly line; customized digital and simulation tools to the way it designs and manufactures products, beyond other technology initiatives, validated through use cases. With Industry 4.0 (I4.0), Embraer has reviewed its industrial strategy, creating "Manufacture Challenge 4.0" as part of its strategic plan.

Adapted to aerospace industry, the I4.0 vision plan aims to verticalize digital and physical systems connections using IIoT enablers, integrating manufacturing systems to have a flexible and reconfigurable smart factory.

Embraer's strategic plan for manufacturing technologies relies on three main pillars: Digital Engineering, Shop Floor and Manufacturing Intelligence (see Fig.1). The Digital Engineering technologies consist of modeling and simulation techniques that allow the creation of the product and process in the digital environment. Furthermore, the Shop Floor technologies materialize the digital model in the real environment with connected physical assets, such as robots and machines. Finally, the Manufacturing Intelligence technologies rely on data analysis and visualization methodologies to extract value from the real environment data and feedback the digital models with information.

This feedback from the real factory to the digital models closes the manufacturing loop, enabling improvement opportunities to be first analyzed and simulated in a virtual environment, and then deployed in the physical factory.

In this scenario, IoT technology is plays a key role in connecting the three pillars, Digital Engineering, Shop Floor and Manufacturing Intelligence and thus making FASTEN an important component in Embraer's portfolio to address the I4.0 challenge [3].

\section{Embraer Use Case}




\subsection{Case Description}

Embraer use case was described at length in [4]. For sake of brevity, the main features and goals are described summarized below.
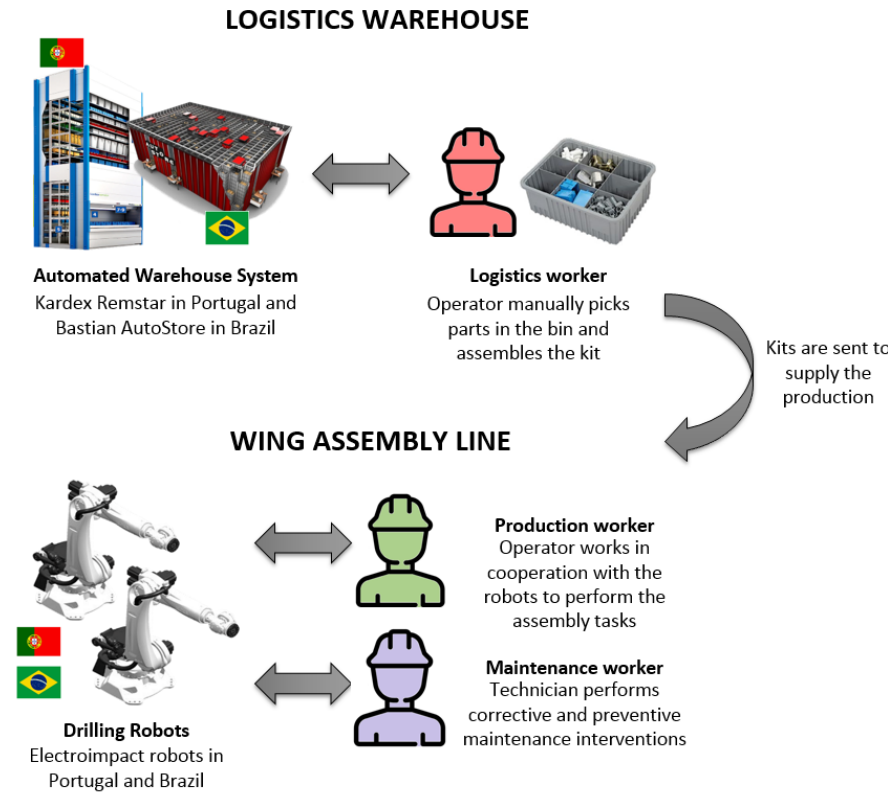

Fig. 2 Embraer Use Case (from [4])

The use case is centered in one of the wing assembly lines of Embraer in Portugal but results and learning's can be transposed to other industrial sites. Three main applications are addressed: an adapting in time automated solution for pick'n'place of parts in automated warehouses (i.e., solving the last human link in the chain, the pick'n'place); improved operation of drilling robots through predictive and prescriptive maintenance and support of decision making in the assembly line, using simulation and optimization tools. These applications are framed, enabled and enhanced using an IoT layer which connects robots, warehouse, corporate systems and humans and should improve upon the following set of challenges:

- Increase manual operations efficiency by the development of a flexible and adaptive solution, kit assembly pick'n'place with an IoT integrated collaborative robotics solution;

- Enhance disruption management of manufacturing operations by integrating - through IoT - machines, humans and computational tools. In specific, enable automatic data collection from robots sensors and event logs for performance monitoring, predictive maintenance and line simulation tools;

- FASTEN IoT integration of operators with physical and digital resources will also enable asset coordination (machine/human/digital) and human-machine collaboration.

\subsection{FASTEN architecture}

Fasten solution architecture is RAMI 4.0 compliant. RAMI 4.00 (Reference Architectural Mode for Industrie 4.0), is a European lead concept model that aligns stakeholders and key players understanding using three main directions: 1) the life cycle value stream (based on IEC standard 62890, it distinguishes between the product model - 
called "type" - and the actual manufactured products - called "instance"); 2) functional layers, from "asset" to "business"; and, 3) the hierarchy levels, developed from IEC 62264, the standard for entreprise-control system integration, they go from Product to the Connected World. By fostering a common language and understanding of the building blocks of the I4.0, interoperable systems and interfaces can be developed and information flows supported. As Brazil has not developed a similar initiative, FASTEN adopted RAMI 4.0 has common reference. FASTEN architecture itself also builds upon FiWARE, a EU funded IoT initiative first originated in the world of smart cities. FiWARE, aims at "build an open sustainable ecosystem around public, royalty-free and implementation-driven software platform standards that will ease the development of new Smart Applications in multiple sectors", address the industrial domain through the Fitman initiative [6].

It was deemed of interest in the project to compare two different implementations of the reference architecture. Besides FiWARE, an Apache lane was developed, using components from the Apache foundation IIoT and developing the necessary connections and integration software. Namely, Apache Kafka, a streaming platform software, was used as foundation for this lane [10].

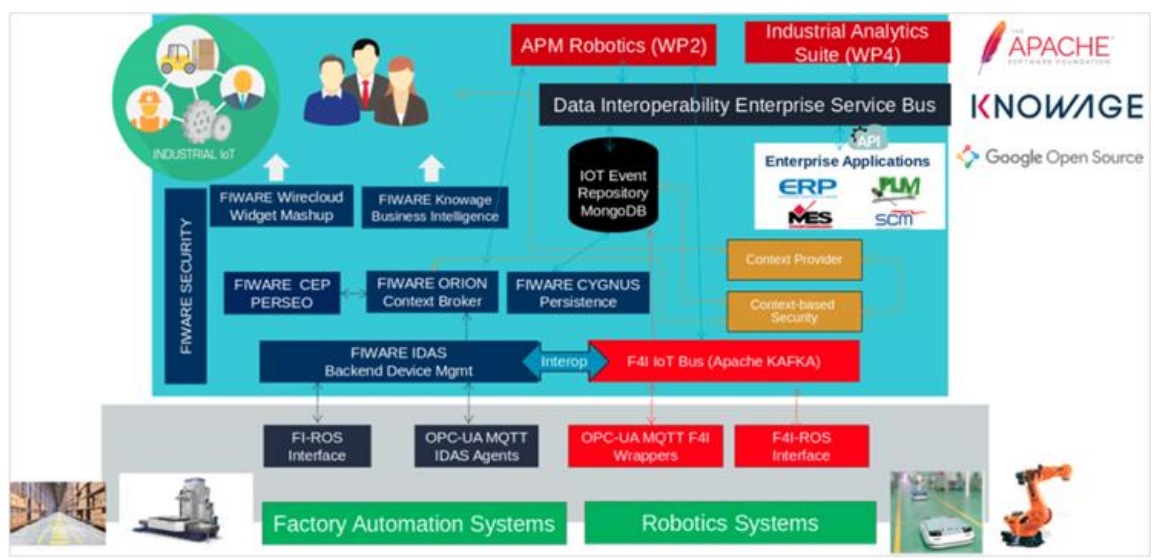

Fig. 4 FASTEN reference architecture (from [4])

With this strategy, FASTEN provides 1) a comparison of implementations from FiWARE and Apache and, 2) develops the necessary software for both lanes to work together, interchangeably.

\subsection{FASTEN IIoT Apache lane}

A description of the Apache lane components and their integration follows. These have been tested, among other, with collection of sensor data and logs from the drilling robots in the wing assembly line at the Embraer plant in Portugal.

The Apache IIoT platform consists of two main components:

VerneMQ Broker is a scalable and distributed high performance MQTT broker which manages the communication between systems and machines. Another component is the Apache Kafka, that provides a high performance and fault tolerant management of all incoming data streams. 


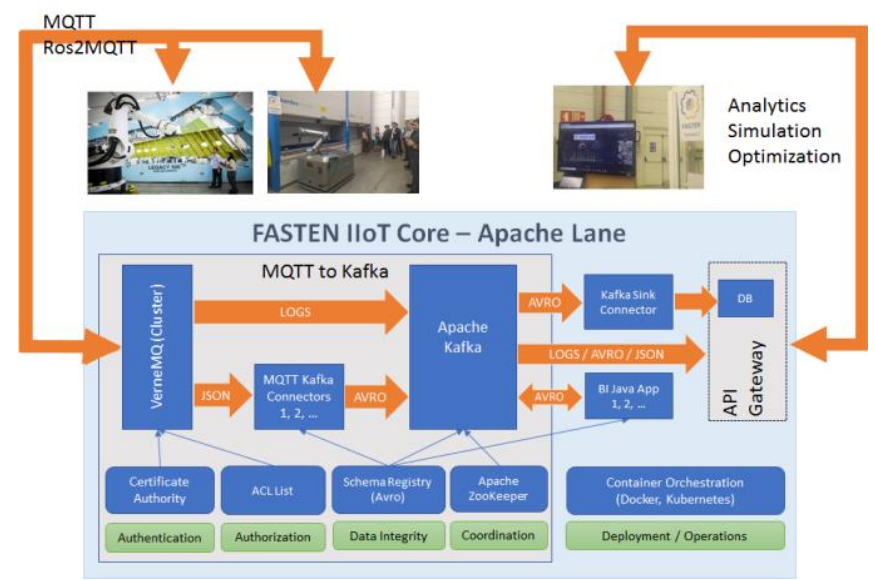

Fig. 5 Apache lane implementation representation, showing components and their application to scenarios of pick'n'place robotics and drilling robot.

In the EMBRAER use case, smart devices like drilling or pick \& place robots or movable manipulators are connected to the IIoT platform via the MQTT broker using MQTT or a Ros2MQTT bridge. The MQTT broker streams the raw IIoT data subscribed to the Kafka topics. For post processing the raw data, Kafka streams it to subscribed complex event processing (CEP) modules. Depending on the requirements and on the configuration, Kafka stores both the raw data as well as the post processed data in a connected Influx data base. All connected systems dedicated to visualization (e.g., dashboard), analytic, simulation or optimization get their data from the data base.

\subsection{Apache lane development results}

Beside the IIoT platform functionalities, another main requirement is an easy installation and configuration in a variety of industrial environments. Therefore a search was done for those open source components better fit to fulfill the industrial requirements set-up in the use case. Where these components where found lacking, new components have been developed. GitHub has been used to store and publish the results of the implementation and integration activities [11]. To ensure the compatibility of the Apache IIoT platform with the client infrastructure, Docker, and Docker Compose, has been used to provide the running environment and the interfaces to the existing systems.

First installations and integrations were done both in Brazil and in Europe - for the different FASTEN use cases - and are providing a very positive feedback in terms of integration and adaptation. In particular, partner INTELIMECH instrumented a drilling robot at the Embraer plant with an external, dedicated vibration and temperature sensor which was able to sustain streamed data to the Apache lane for pre-processing and visualization.

\subsection{FASTEN Suite Tool}

The IIoT of machines, devices, systems and humans enables the creation of valuable high level services. These take advantage of accessing historical data (i.e., past system dynamics and events), real time signals (current events and monitoring of deviations from predicted behavior) and can thus coordinate entities to deal with forecasted problems or speed up reaction upon real time events. The FASTEN Suite Tool is an example of this, a set of tools for decision support in the assembly line: from load balancing to product 
modifications, to maintenance management, by recourse to predictive and prescriptive analytics. A brief overview of the FASTEN Suite current capabilities is given and a more in-depth description is presented in the companion paper [5].

The FASTEN Suite has 3 modules: the Simulator-Optimizer, the Predictive and the Prescriptive Analytic and Real-Time Monitoring. The first uses a combination of discrete event simulation, and linear programming and heuristics to solve problems such as: optimize the production layout, allocation of resources and time to execute maintenance activities, and measure the impact of making such decisions in the system's performance. The second uses open source machine-learning libraries to analyze data from the robotic resources and predict the probability of failure of these resources. Finally, the third combines data visualization tools to present the results obtained by the other modules to the end user, as well as, real time data generated by the sensors and equipments presented in the shop floor. The integration between these modules is possible by the FASTEN IIoT platform, that enables communication and data exchange between them. Additionally, the IIoT Platform allows data retrieval from corporate systems, such as the Manufacturing Execution System (MES) and Maintenance Management System (MMS), to be used by the suite modules. This Suite enables companies to make decisions faster in situations like production resources failures; to increase predictive maintenance activities and thus reducing corrective actions; and optimizing assembly lines layout and resources allocation. Real-time monitoring of the different levels of information - real-time and processed - is made possible using tailored dashboards. These capabilities result in fewer stoppages and maintenance costs and an increase in the system's productivity.

For this phase of the project, developments focused in implementing the ground work to support the predictive algorithms, such as connections to corporate systems and drilling robots to allow data recovery (e.g. alarms and process data generated by drilling robots, temperature and vibration real time data, collected from robots). In the FASTEN IIoT platform, the first module is capable of resource optimization, e.g. allocating man-hour in the production activities and considering the available parts, having the increasing of productivity as objective; and to evaluate different scenarios generated by optimization and to provide expected information. The second module allows analyzing the acquired data behavior by a non-supervised model to monitor resources availability (drilling robots health). Finally, in the third module, information processed by the prior Suite modules is presented in dashboards augmenting processes visibility. With these capabilities, the following scenario was tested: the production resources continually send data to the IIoT Platform. The second module gets and analyzes the equipment data to predict the probability of the resources failing, while the third module displays that probability and the most relevant information. When such probability exceeds a threshold, an alarm is triggered and the first module is activated in order to estimate the best time to execute the maintenance operations, based on the production and the maintenance system information. In addition, it evaluates the impact on the system of making such a decision. Finally, the results are displayed on dashboards in the third module.

\subsection{FASTEN Robotic System}

The FASTEN robotic system is constituted by a collaborative mobile manipulator, i.e. an autonomous guided vehicle (AGV) equipped with an omnidirectional driving system, capable of moving the robot in any direction in an efficient manner, and a collaborative robotic manipulator, to promote co-existence and co-working with human operators in the same industrial environment (see Fig. 7). One of the key features of the FASTEN robotic system is the ability to successfully recognize, grasp, and handle a multitude of parts stored in an automated warehouse, thus enhancing the efficiency in intra-logistics operations. 
Additionally, special emphasis is being applied to ensure that the robot can easily be configured to handle novel objects, and, more ambitiously, autonomously handle new parts.

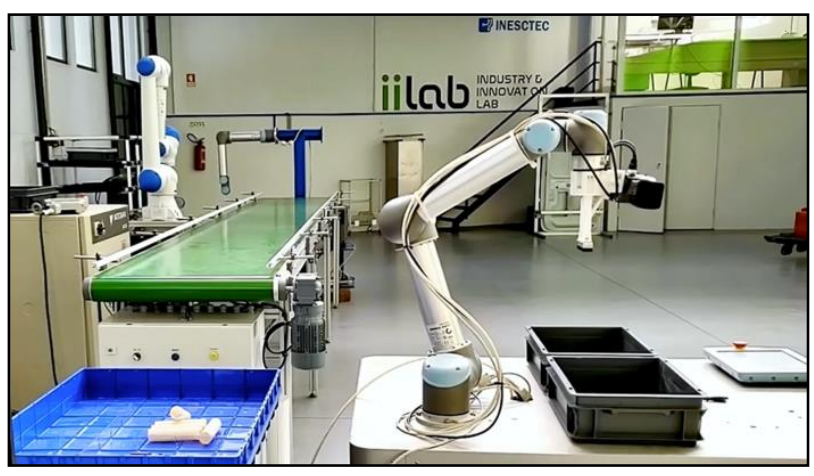

Fig. 7 Robotic pick'n'place solution at INESCTEC
In what regards its underlying controlling software, the FASTEN robotic system is fully integrated into the Open Scalable Production System (OSPS), a set of software modules tailored to advocate the assimilation of production resources (robotic systems, automation equipment, human operators) into the Digital Manufacturing paradigm. On

the robotics scope, the OSPS software architecture can be divided into two domains: serverlevel and local-level software modules. On the server-side, the Advanced Plant Model (APM) [7], responsible for keeping a Digital Twin representation of the industrial environment, and the Production Manager (PM), which is responsible for issuing and coordinating the execution of manufacturing or logistics tasks by the production resources, are the most important modules. On what regards software running on the robotic system (local level), the Task Manager (TM) is the entity responsible for orchestrating the execution of manufacturing or logistic tasks into a set of pre-programmed robotic skills, which correspond to encapsulated Robot Operating System (ROS) software modules for achieving a specific and bounded function, as is example the Locate Skill, responsible for selecting, recognizing, and precisely locating the most appropriate object to be grasped.

Besides the responsibility of ensuring the successful execution of manufacturing or logistics tasks, the OSPS also promotes the output of a set of performance indicators to the IoT platform. The FASTEN robotic system developed for the Embraer use case is currently capable of outputting its location on the warehouse, battery level, Wi-Fi connectivity, processor and memory load of its internal industrial computer, current task status, amongst other data. Furthermore, the OSPS software stack is currently being applied in a multitude of platforms for different R\&D projects, such as the complementary use case of the FASTEN project, focused on a mobile manipulator for machine tending in an additive manufacturing industrial environment, further detailed in [8].

\section{FASTEN EU-Brazil: exploitation \& dissemination}

To achieve the results aimed by FASTEN, a considerable effort in collaborative work has been put into practice. This is been fostered by both Project coordinators, namely INESCTEC, from the EU side and INESC P\&D BRASIL from Brazil. Also key is both the presence of its industrial driving companies, Embraer Portugal and Embraer SA, and ThyssenKrupp (in Brazil), which belong to groups with footprints in both Brazil and Europe. Also a set of research and private companies that develop the FASTEN reference solution spanning both regions and work together. From the EU side we have INTELLIMECH, PACE \& TXT, and Politecnico di Milano; and from the Brazilian side PUCRS, UFBA, UFSC, UFG, and Bradel. These partners bring different expertise's and know-hows that combined are nurturing FASTEN results.

The interrelationship activities between EU and Brazilian research teams can be seen insofar in about 10 scientific publications, with topics covering: a) FASTEN Holistic 
Simulation-optimization tool, b) FASTEN Predictive and Prescriptive Tool, c) Real-Time Monitoring, d) Grasping system, e) FASTEN IIoT, f) Automation; g) Industry 4.0 architecture; h) Predictive maintenance; i) Simulation, j) Navigation of Mobile Robots, k) Decision-making regarding the deployment of productive resources (3D printers) in locations of a spare parts supply chains. Moreover, a series of new joint publications are in development approaching the latest developments considering all pillars of the project.

In particular regarding the Embraer use case, it was driven by a mix team from Portugal and Brazil and its requirements are transversal to the different units of the group. As such, testing the deployments of the FASTEN IIoT platform also in Brazil is seen has desirable and is under discussion. In terms of collaboration and sustainability, one practice in the project regards the engagement of the team to develop solutions with minimum environmental impact: several tests have been conducted by means of using simple positive environmental solutions, for instance, pick and pack robotic operations based on compressed air, instead of oil hydraulic systems. The FASTEN exploitation plan to further explore the assets generated in the project, representing a tangible initiative to the further usage of the technologies developed.

\section{Final comments}

FASTEN is across $2 / 3$ of its duration and has brought already mid-term results for demonstration into one of the Embraer plants for assessment in the operational scenario.

Namely it has already:

1) Produced a set of IIoT components, contributing to both FiWARE and Apache IoT ecosystem. These components are interoperable and were already deployed to connect robots, corporate emulated systems, and analytics and simulation decision support tools;

2) Developed and tested an automation solution for pick'n'place, using a skills based approach, and did a first assessment at Embraer plants using 3D printed parts, analogous to real aircraft parts;

3) Developed assembly line and optimization simulation solutions, as also a first foray of predictive analytics solution, currently being further developed using robot sensor data, process and alarm logs, as also historical maintenance data.

A final pilot iteration in the operational setting will follow to test the final iteration of the solutions developed. Besides the specific technical developments, FASTEN further aims at laying out the seeds of further cooperation between Europe and Brazil regarding Industry 4.0, disseminating the developed IIoT components and seeking to enlarge the research and industry community that could benefit from its exploitation. As such it encourages further contacts through its website at http://fastenmanufacturing.eu.

In sum, it can be said that aeronautics faces global challenges of sustainability and efficiency, where global industrial footprints and interlaced supply chains are the reality. With Horizon Europe looming ahead, FASTEN brings a positive case study on industrial Europe-Brazil collaboration, that could be leveraged to strengthen further joint developments for common gains between Europe and Brazil.

This work was supported by H2020, project grant 777096, FASTEN - Flexible and Autonomous Manufacturing Systems for Custom-Designed Products.

\section{References}

1. H. Kaggermann, W. Wahlster, J. Helbig, Recommendations for implementing the strategic initiative INDUSTRIE 4.0, (2013) 
2. S. Wang, J. Wan, D. Li, C. Zhang, Implementing Smart Factory of Industrie 4.0: An Outlook. International Journal of Distributed Sensor Networks, vol 2016, 2015

3. A, B. Carmesini. Developing Advanced Manufacturing in the Aerospace Industry: Embraer's Success Cases. 22o Seminário Internacional de Alta Tecnologia, 2017

4. R. Reis, F. Diniz, L. Mizioka, P. Olivio, G. Lemos, M. Quintiães, R. Menezes, F. Amadio, N. Caldas, "FASTEN: an IoT plataform for manufaturing. Embraer use case", 8th EASN, (2018)

5. R. Vita, N. Caldas, J. Basto, S. Alcalá, and F. Diniz, “An IIoT-based architecture for decision support in the aeronautic industry", 9th EASN, (to be published)

6. Fitman: http://www.fiware4industry.com/?page_id=290

7. C. Toscano, F. Arrais, G. Veiga (2017, November). Enhancement of industrial logistic systems with semantic $3 \mathrm{~d}$ representations for mobile manipulators. In Iberian Robotics conference (pp. 617-628). Springer, Cham.

8. R. Arrais, G. Veiga, T. Ribeiro, D. Oliveira, R. Fernandes, A. Conceição, G. S., \& P. Farias, C. M. A. (2019, September). Application of the Open Scalable Production System to Machine Tending of Additive Manufacturing Operations by a Mobile Manipulator. In EPIA Conference on Artificial Intelligence (pp. 345-356). Springer, Cham.

9. Reference Architecture Model Industrie 4.0 (RAMI4.0), Status Report, VDI, 2015

10. https://kafka.apache.org 